\title{
Studies on the Constituents of Brazilian Propolis. II $^{1)}$
}

\author{
Shigemi Tazawa, Tsutomu Warashina, and Tadataka Noro* \\ Graduate School of Nutritional and Environmental Sciences, University of Shizuoka, 52-1 Yada, Shizuoka 422-8526, \\ Japan. Received April 23, 1999; accepted June 29, 1999
}

\begin{abstract}
Seven new $p$-coumaric acid derivatives along with seventeen known compounds, including four flavonoids, one prenylated phenolic acid, four diterpenoic acids, one lignan, two $p$-coumaric acid esters and five cinnamic acid derivatives, were isolated from the ethyl acetate soluble fraction of $75 \%$ ethanol extract of Brazilian propolis. New compounds were elucidated as $(E)-2,3-d i h y d r o c o n i f e r y l ~ p-c o u m a r a t e, ~(E)-3-\{2,3-d i h y d r o-2-[2-[(E)-p-$

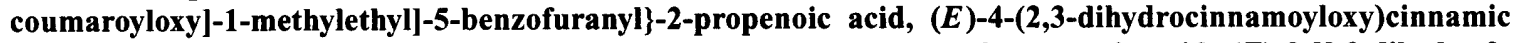
acid, $(E)$-3-(2,2-dimethyl-3,4-dihydro-3-hydroxy-2H-1-benzopyran-6-yl)-2-propenoic acid, $(E)$-3-[2,3-dihydro-2(1-methylethenyl)-5-benzofuranyl]-2-propenoic acid, $(E)$-3-[2,3-dihydro-2-(1-methylethenyl)-7-prenyl-5-benzofu-

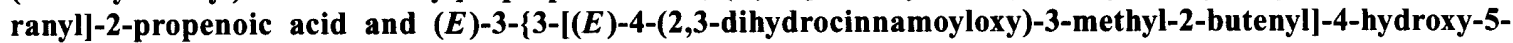
prenylphenyl\}-2-propenoic acid, on the basis of spectral evidence and chemical reaction. Five compounds: dihydrokaempferol (aromadendrin), 6-methoxykaempferol, 4-hydroxy-3-prenylbenzoic acid, plicatin B and capillartemisin A, were isolated from propolis for the first time.
\end{abstract}

Key words propolis; Apis mellifera; p-coumaric acid derivative; diterpenoic acid; flavonoid; Brazil

Propolis (bee glue) is a resinous hive product gathered by honeybees (Apis mellifera) from the buds and bark of certain trees and plants. We previously reported on the isolation and characterization of prenylcinnamic acid derivatives from Brazilian propolis. ${ }^{1)}$ In this paper, we describe the isolation and structural elucidation of 24 constituents, including seven new p-coumaric acid derivatives from Brazilian propolis.

\section{Results and Discussion}

Propolis, which was obtained from the state of Minas Gerais in Brazil, was extracted with $75 \%$ ethanol at room temperature. The extract was processed by the method described in the Experimental section. Twenty-four compounds were isolated from the ethyl acetate soluble fraction of the $75 \%$ ethanol extract of Brazilian propolis.

Compounds $1-4$ were known flavonoids. They were identified as naringenin ${ }^{2,3)}(1)$, sakuranetin ${ }^{3)}(2)$, dihydrokaempferol (aromadendrin) ${ }^{3,4)}(3)$ and 6-methoxykaempferol ${ }^{5)}$ (4). Compound 5 was a known phenolic compound. It was identified as 4-hydroxy-3-prenylbenzoic $\operatorname{acid}^{6)}(5)$. Compounds $6-9$ were known diterpenoic acids. They were identified as 15-acetoxyisocupressic acid $^{7)}(6)$, agathic acid 15methyl ester ${ }^{8)}(7)$, communic acid ${ }^{7)}(8)$ and dehydroabietic $\operatorname{acid}^{9)}(9)$. Compound 10 was a lignan of a trimeric coniferyl alcohol. It was identified as 1-(4-hydroxy-3-methoxyphenyl)1,2-bis $\{4-[(E)-3$-acetoxypropen-1-yl]-2-methoxyphenoxy $\}-$ propan-3-ol acetate ${ }^{10)}(\mathbf{1 0})$. Compounds 11 and 12 were known $p$-coumaric acid esters. They were identified as benzyl $p$-coumarate (11) and phenethyl $p$-coumarate (12). Compounds 13-17 were known cinnamic acid derivatives. They were identified as cinnamic acid (13), ferulic acid (14), 3(2,2-dimethy-2H-1-benzopyran-6-yl)-2-propenoic acid ${ }^{11)}(15)$, plicatin $B^{12)}(16)$ and capillartemisin $A^{13)}(17)$. These known compounds were identified by comparison of spectral data and specific optical rotation with values in the literature and/or authentic samples. This is the first report of the isolation of 3, 4, 5, 16 and 17 from propolis.

Compound 18 was obtained as an amorphous powder and afforded a $[\mathrm{M}+\mathrm{H}]^{+}$ion peak at $m / z 329\left(\mathrm{C}_{19} \mathrm{H}_{21} \mathrm{O}_{5}\right)$ in the positive FAB-MS. The IR spectrum of 18 showed a signal at
$1688 \mathrm{~cm}^{-1}$. The ${ }^{1} \mathrm{H}-\mathrm{NMR}$ spectrum displayed signals due to a dihydroconiferyl group $[\delta 1.98(2 \mathrm{H}, \mathrm{dt}, J=7.5,6.5 \mathrm{~Hz})$, $2.66(2 \mathrm{H}, \mathrm{t}, J=7.5 \mathrm{~Hz}), 3.83(3 \mathrm{H}, \mathrm{s}), 4.16(2 \mathrm{H}, \mathrm{t}, J=6.5 \mathrm{~Hz})$, $6.64(1 \mathrm{H}, \mathrm{dd}, J=8.5,1.5 \mathrm{~Hz}), 6.71(1 \mathrm{H}, \mathrm{d}, J=8.5 \mathrm{~Hz}), 6.78$ $(1 \mathrm{H}, \mathrm{d}, J=1.5 \mathrm{~Hz})]$ except for those of the $p$-coumaric acid moiety. Assignment of the signals in the ${ }^{1} \mathrm{H}$ - and ${ }^{13} \mathrm{C}-\mathrm{NMR}$ spectra was confirmed based on ${ }^{1} \mathrm{H}-{ }^{1} \mathrm{H}$ shift correlation spectroscopy (COSY) and ${ }^{1} \mathrm{H}-{ }^{13} \mathrm{C}$ COSY spectral data. In the heteronuclear multiple-bond correlation (HMBC) spectrum, the oxygenated methylene signal $\delta 4.16\left(\mathrm{H}-9^{\prime}\right)$ showed correlation with the carboxyl carbon signal at $\delta 168.6$ (C-9). Therefore, the structure of $\mathbf{1 8}$ was deduced to be $(E)-2,3-\mathrm{di}-$ hydroconiferyl $p$-coumarate.

Compound 19 was obtained as an amorphous powder and afforded a [M] ${ }^{+}$ion peak at $m / z 394\left(\mathrm{C}_{23} \mathrm{H}_{22} \mathrm{O}_{6}\right)$ in the positive FAB-MS. The IR spectrum of 19 showed signals at 1688 and $1682 \mathrm{~cm}^{-1}$. The ${ }^{1} \mathrm{H}$ - and ${ }^{13} \mathrm{C}-\mathrm{NMR}$ spectra, and the ${ }^{1} \mathrm{H}-$ ${ }^{1} \mathrm{H}$ and ${ }^{1} \mathrm{H}-{ }^{13} \mathrm{C}$ COSY spectral data revealed signals due to a $p$-coumaric acid moiety and agreed with those of 18 . The ${ }^{1} \mathrm{H}-\mathrm{NMR}$ and ${ }^{1} \mathrm{H}-{ }^{1} \mathrm{H}$ COSY spectrum of 19 exhibited one methyl $[\delta 1.05(3 \mathrm{H}, \mathrm{d}, J=7.0 \mathrm{~Hz})]$, one methine $[\delta 2.23(1 \mathrm{H}$, $\mathrm{m})]$, one methylene $[\delta 3.11(1 \mathrm{H}$, dd, $J=16.0,8.0 \mathrm{~Hz})$ and $3.35(1 \mathrm{H}, \mathrm{dd}, J=16.0,4.4 \mathrm{~Hz})]$, one oxygenated methylene $[\delta$ $4.22(2 \mathrm{H}, \mathrm{m})]$, one oxygenated methine $[\delta 4.85(1 \mathrm{H}, \mathrm{m})]$, two olefins $[\delta 6.30(1 \mathrm{H}, \mathrm{d}, J=16.0 \mathrm{~Hz}), 7.56(1 \mathrm{H}, \mathrm{d}, J=16.0$ $\mathrm{Hz})$ and $\delta 6.33(1 \mathrm{H}, \mathrm{d}, J=16.0 \mathrm{~Hz}), 7.61(1 \mathrm{H}, \mathrm{d}, J=16.0$ $\mathrm{Hz})]$, one aromatic $\mathrm{ABX}$ system $[\delta 6.74(1 \mathrm{H}, \mathrm{d}, J=8.5 \mathrm{~Hz})$, $7.32(1 \mathrm{H}$, br d, $J=8.5 \mathrm{~Hz}), 7.47(1 \mathrm{H}$, br s $)]$ and one aromatic $\mathrm{A}_{2} \mathrm{~B}_{2}$ system $[\delta 6.81(2 \mathrm{H}, \mathrm{d}, J=8.5 \mathrm{~Hz}), 7.46(2 \mathrm{H}, \mathrm{d}, J=8.5$ $\mathrm{Hz})]$. In the HMBC spectrum, the signal at $\delta 4.22(\mathrm{H}-12)$ showed correlation with the carboxyl carbon signals at $\delta$ 169.2 (C-9'), so the ester is made with carboxylic acid of $p$ coumaric acid. Other long-range correlations, depicted in Fig. 1 by arrows, indicated the presence of a $p$-coumaric acid moiety and a dihydrobenzofuran ring. Consequently, the structure of 19 was deduced to be $(E)-3-\{2,3-$ dihydro-2-[2$[(E)$-p-coumaroyloxy]-1-methylethyl]-5-benzofuranyl $\}-2$ propenoic acid. The configurations of $\mathrm{C}-2$ and $\mathrm{C}-11$ were not determined.

Compound 20 was obtained as an amorphous powder and 
<smiles>COc1cc(CCCOC(=O)/C=C/c2ccc(O)cc2)ccc1O</smiles>

18<smiles>CC(COC(=O)/C=C/c1ccc(O)cc1)C1Cc2cc(/C=C/C(=O)O)ccc2O1</smiles><smiles>O=C(O)/C=C/c1ccc(OC(=O)CCc2ccccc2)cc1</smiles><smiles>CC1(C)Oc2ccc(/C=C/C(=O)O)cc2CC1O</smiles><smiles>C=C(C)C1Cc2cc(/C=C/C(=O)O)ccc2O1</smiles>

22<smiles>C=C(C)C1Cc2cc(/C=C/C(=O)O)cc(CC=C(C)C)c2O1</smiles><smiles>CC(C)=CCc1cc(/C=C/C(=O)O)cc(C/C=C(\C)COC(=O)CCc2ccccc2)c1O</smiles>

Chart 1

afforded a $[\mathrm{M}+\mathrm{H}]^{+}$ion peak at $m / z 297\left(\mathrm{C}_{18} \mathrm{H}_{17} \mathrm{O}_{4}\right)$ in the positive FAB-MS. The IR spectra of 20 showed signals at 1757 and $1686 \mathrm{~cm}^{-1}$. The ${ }^{1} \mathrm{H}$ - and ${ }^{13} \mathrm{C}-\mathrm{NMR}$ and the ${ }^{1} \mathrm{H}-{ }^{1} \mathrm{H}$ and ${ }^{1} \mathrm{H}-{ }^{13} \mathrm{C}$ COSY spectral data showed the presence of one dihydrocinnamoyl group and one $p$-coumaric acid moiety. In the HMBC spectrum, the signal at $\delta 2.90\left(\mathrm{H}-8^{\prime}\right)$ showed a correlation with the carbon signals at $\delta 139.9$ which was assigned to $\mathrm{C}-1^{\prime}$ of the dihydrocinnamoyl group, not the $p$-oxybenzene group. The possibility of a dihydro- $p$-coumaric acid moiety was denied. This structure was determined by comparison with the data of $p$-coumaric acid and $(E)$-3-prenyl-4(2,3-dihydrocinnamoyloxy)cinnamic acid, ${ }^{1,14)}$ which attached a prenyl group to $\mathbf{2 0}$. Thus, the structure of $\mathbf{2 0}$ was concluded to be (E)-4-(2,3-dihydrocinnamoyloxy)cinnamic acid.

Compound 21 was obtained as an amorphous powder and afforded a [M] ${ }^{+}$ion peak at $m / z 248\left(\mathrm{C}_{14} \mathrm{H}_{16} \mathrm{O}_{4}\right)$ in the positive FAB-MS. The IR spectrum of 21 showed a signal at $1682 \mathrm{~cm}^{-1}$. The ${ }^{1} \mathrm{H}-\mathrm{NMR}$ spectrum indicated the presence of two tertiary methyls $[\delta 1.26(3 \mathrm{H}, \mathrm{s}), 1.34(3 \mathrm{H}, \mathrm{s})]$, one methylene $[\delta 2.75(1 \mathrm{H}$, dd, $J=16.5,7.0 \mathrm{~Hz}), 3.04(1 \mathrm{H}, \mathrm{dd}, J=$ $16.5,5.0 \mathrm{~Hz})$, one hydroxy methine $[\delta 3.78(1 \mathrm{H}, \mathrm{dd}, J=7.0$, $5.0 \mathrm{~Hz})]$, one propenoic acid group $[\delta 6.30(1 \mathrm{H}, \mathrm{d}, J=16.0$ $\mathrm{Hz}), \delta 7.58(1 \mathrm{H}, \mathrm{d}, J=16.0 \mathrm{~Hz})]$ and one aromatic ABX sys- tem $[\delta 6.76(1 \mathrm{H}, \mathrm{d}, J=8.0 \mathrm{~Hz}), 7.32(1 \mathrm{H}, \mathrm{br} \mathrm{s}), 7.34(1 \mathrm{H}$, br d, $J=8.0 \mathrm{~Hz}$ )]. In the HMBC spectrum, the signal at $\delta 3.78$ (H-3) showed a correlation with two tertiary methyl carbon signals at $\delta 25.9(\mathrm{C}-12)$ and $21.4(\mathrm{C}-13)$, and with one aromatic carbon at $\delta 121.8(\mathrm{C}-4 \mathrm{a})$. In addition, the aromatic proton signal at $\delta 7.32(\mathrm{H}-5)$ correlated with the methylene carbon signal at $\delta 31.9(\mathrm{C}-4)$ and $146.4(\mathrm{C}-9)$. Therefore, the position of the hydroxyl group in $\mathbf{2 1}$ was determined to be $\mathrm{C}$ 3. Other long-range correlations indicated the presence of a dihydrobenzopyran ring (Fig. 2). This structure was also supported by comparison with the data of $(E)$-3-(2,2-dimethyl3,4-dihydro-3-hydroxy-8-prenyl-2H-1-benzopyran-6-yl)-2propenoic acid. ${ }^{1,8)}$ Consequently, the structure of 21 was deduced to be $(E)$-3-(2,2-dimethyl-3,4-dihydro-3-hydroxy-2H1-benzopyran-6-yl)-2-propenoic acid.

Compound 22 was obtained as an amorphous powder and afforded a $[\mathrm{M}+\mathrm{H}]^{+}$ion peak at $m / z 231\left(\mathrm{C}_{14} \mathrm{H}_{15} \mathrm{O}_{3}\right)$ in the positive FAB-MS. The IR spectrum of 22 showed a signal at $1682 \mathrm{~cm}^{-1}$. Based on the ${ }^{1} \mathrm{H}$ - and ${ }^{13} \mathrm{C}$-NMR spectra, 22 was determined to be a cinnamic acid derivative. The ${ }^{1} \mathrm{H}-\mathrm{NMR}$ spectrum of 22 revealed one oxygenated methine [ $\delta 4.35$ $(1 \mathrm{H}$, dd, $J=8.0,4.5 \mathrm{~Hz})]$, one methylene $[\delta 2.78(1 \mathrm{H}$, dd, $J=13.5,8.0 \mathrm{~Hz}), 2.91(1 \mathrm{H}, \mathrm{dd}, J=13.5,4.5 \mathrm{~Hz})]$, and a set of 


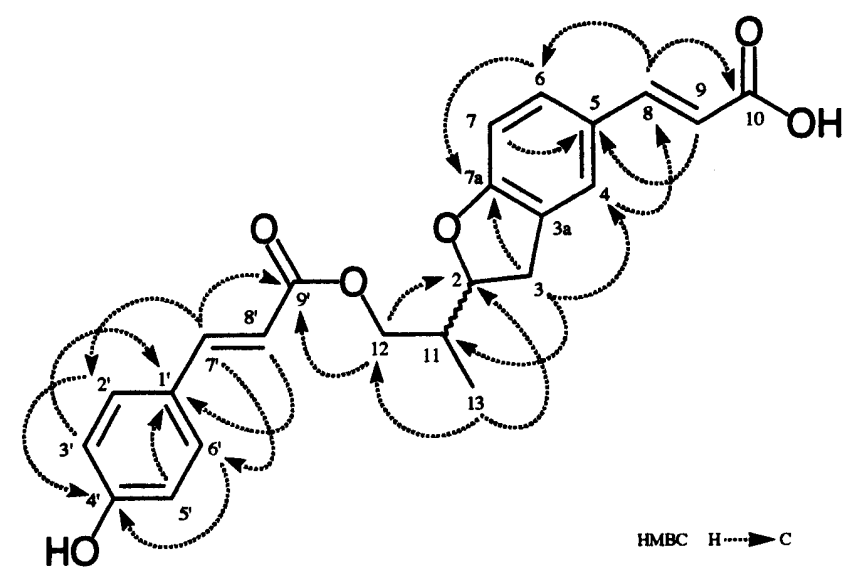

Fig. 1. HMBC Correlation of $\mathbf{1 9}$

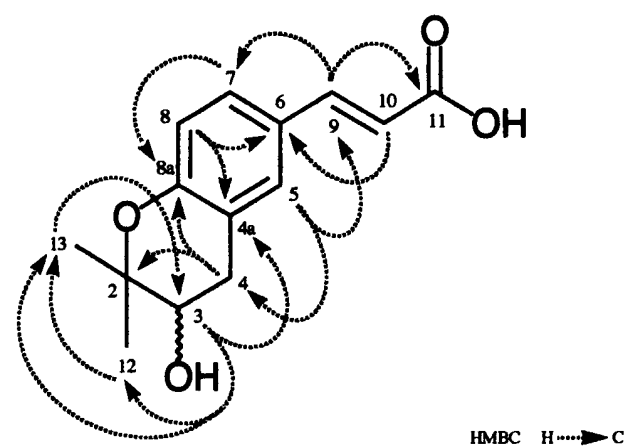

Fig. 2. HMBC Correlation of $\mathbf{2 1}$

isopropenyl signals [ $\delta 1.79(3 \mathrm{H}, \mathrm{s}), 4.77(1 \mathrm{H}, \mathrm{s}), 4.85(1 \mathrm{H}$, s)]. In the HMBC spectrum, the signal at $\delta 4.35(\mathrm{H}-2)$ showed correlation with the carbon signals at $\delta 18.1$ (C-13), $111.3(\mathrm{C}-12)$ and $127.6(\mathrm{C}-3 \mathrm{a})$. The aromatic proton signal at $\delta 7.34(\mathrm{H}-4)$ correlated with the methylene carbon signal at $\delta$ 38.1 (C-3) (Fig. 3).

Methylation of 22 with diazomethane furnished the methyl ester (22a). Compound 22a afforded a $[\mathrm{M}+\mathrm{H}]^{+}$ion peak at $m / z 245\left(\mathrm{C}_{15} \mathrm{H}_{17} \mathrm{O}_{3}\right)$ in the positive FAB-MS. The IR spectrum, $1688 \mathrm{~cm}^{-1}$, suggested the ester of 22 . $22 \mathrm{a}$ was in agreement with the NMR data of the (E)-3-[2,3-dihydro-2(1-methylethenyl)-5-benzofuranyl]-2-propenoic acid methyl ester which was previously isolated from Baccharis linearis. ${ }^{11)}$ Based on these facts, the structure of 22 was deduced to be $(E)-3$-[2,3-dihydro-2-(1-methylethenyl)-5-benzofuranyl]-2-propenoic acid.

Compound 23 was obtained as an amorphous powder and afforded a $[\mathrm{M}+\mathrm{H}]^{+}$ion peak at $m / z 299\left(\mathrm{C}_{19} \mathrm{H}_{23} \mathrm{O}_{3}\right)$ which was 68 mass units larger than that of 22 in the positive FABMS. The IR spectrum of 23 showed a signal at $1682 \mathrm{~cm}^{-1}$. The ${ }^{1} \mathrm{H}$ - and ${ }^{13} \mathrm{C}$-NMR spectra exhibited signals similar to those of 22. The ${ }^{1} \mathrm{H}-\mathrm{NMR}$ spectrum revealed a set of prenyl signals [ $\delta 1.73(3 \mathrm{H}, \mathrm{s}), 1.78(3 \mathrm{H}, \mathrm{s}), 3.36(2 \mathrm{H}, \mathrm{d}, J=7.0 \mathrm{~Hz})$, $5.33(1 \mathrm{H}, \mathrm{br} \mathrm{t})]$ and two aromatic signals $[\delta 7.11(1 \mathrm{H}, \mathrm{br} \mathrm{s})$, $7.25(1 \mathrm{H}, \mathrm{br} \mathrm{s})]$. The structure of 23 was determined by analysis of its ${ }^{1} \mathrm{H}-{ }^{1} \mathrm{H}$ and ${ }^{1} \mathrm{H}-{ }^{13} \mathrm{C}$ COSY spectrum and HMBC spectrum, and by comparison with the data of 22 and 22a. ${ }^{11)}$ In the HMBC spectrum of 23, the proton signal at $\delta$ $7.68(\mathrm{H}-8)$ showed correlation with the aromatic carbon signals at $\delta 128.9$ (C-6) and $129.8(\mathrm{C}-4)$. So, the prenyl moiety is attached at the 7 position. Other long-range correlations

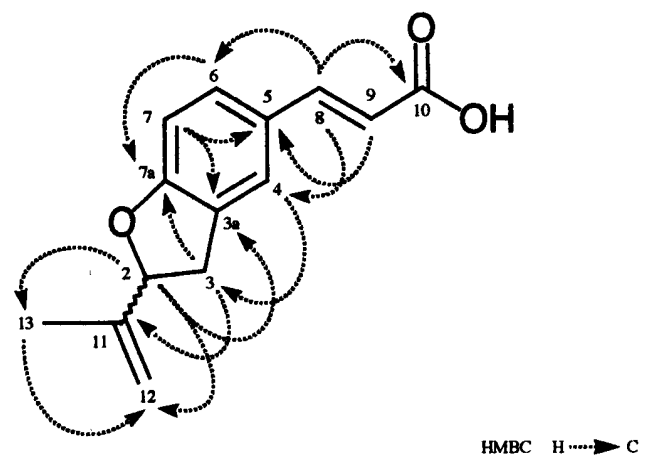

Fig. 3. HMBC Correlation of $\mathbf{2 2}$

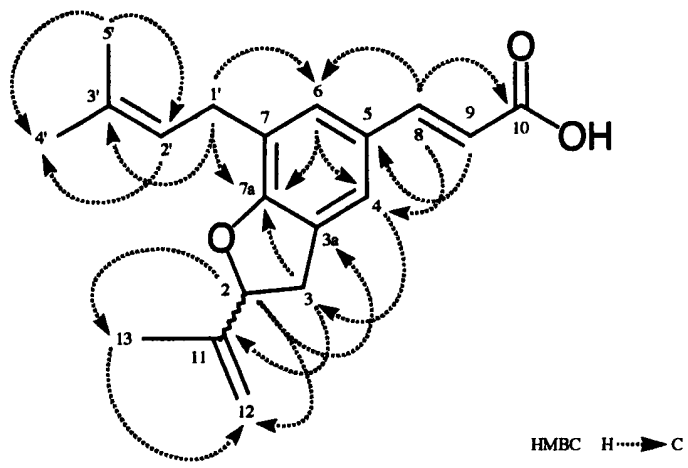

Fig. 4. HMBC Correlation of 23

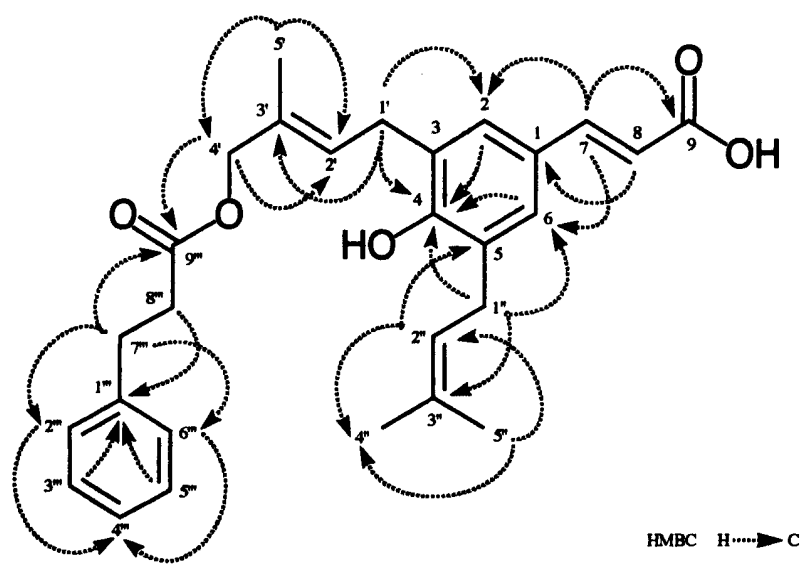

Fig. 5. HMBC Correlation of $\mathbf{2 4}$

are depicted in Fig. 4. Consequently, the structure of 23 was concluded to be (E)-3-[2,3-dihydro-2-(1-methylethenyl)-7prenyl-5-benzofuranyl]-2-propenoic acid.

Compound 24 was obtained as an amorphous powder and afforded a $[\mathrm{M}-\mathrm{H}]^{+}$ion peak at $m / z 447\left(\mathrm{C}_{28} \mathrm{H}_{31} \mathrm{O}_{5}\right)$ in the positive FAB-MS. The IR spectrum of 24 showed signals at 1720 and $1686 \mathrm{~cm}^{-1}$. The ${ }^{1} \mathrm{H}$ - and ${ }^{13} \mathrm{C}$-NMR spectra exhibited signals similar to those of capillartemisin $A^{13)}(17)$, except for the presence of a dihydrocinnamoyl moiety. ${ }^{1,14)}$ Capillartemisin $\mathrm{A}$ is the $E$-hydroxyisoprenyl type, while capillartemisin $\mathrm{B}^{13)}$ is the $Z$ type. So, 24 is the $E$ type. In the HMBC spectrum, the oxygenated methylene proton signal at $\delta 4.52\left(\mathrm{H}-4^{\prime}\right)$ showed a correlation with the carboxyl carbon signal at $\delta 172.8\left(\mathrm{C}-9^{\prime \prime \prime}\right)$. Other long-range correlations, depicted in Fig. 5 by arrows, supported the presence of a dihydrocinnamoyl group and capillartemisin A. Thus, the structure of 24 was deduced to be $(E)-3-\{4$-hydroxy-3-[(E)-4- 
(2,3-dihydrocinnamoyloxy)-3-methyl-2-butenyl]-5-prenylphenyl\}-2-propenoic acid.

In this study, we found that the isolated compounds $1,2,9$, 15,16 and the methyl ester of 22 were contained in different Baccharis species (Compositae) ${ }^{11,16)}$ which have been widely used as a folk medicine in Brazil. The genus Baccharis is widespread in the tropical South American zone, and has many known constituents, including phenolic compounds, flavonoids and diterpenes. Thus, the phytochemical investigation suggested that Baccharis species are a significant sources of propolis.

\section{Experimental}

Optical rotations were determined with a JASCO DIP-1000 digital polarimeter. UV spectra were measured on a Beckman DU 640 spectrophotometer. IR spectra were measured on a JASCO FT/IR-230 fourier transform IR spectrometer. FAB-MS spectra were taken on a JEOL JMS-SX102 spectrometer. ${ }^{1} \mathrm{H}-$ and ${ }^{13} \mathrm{C}$-NMR were recorded on a JEOL GSX-500 (500 and $125.65 \mathrm{MHz}$, respectively). Chemical shifts are given on a $\delta(\mathrm{ppm})$ scale with tetramethylsilane (TMS) as an internal standard. The ${ }^{1} \mathrm{H}-{ }^{1} \mathrm{H}$ COSY, ${ }^{1} \mathrm{H}-{ }^{13} \mathrm{C}$ COSY and $\mathrm{HMBC}$ spectra were recorded with standard JEOL software. HPLC was run on JASCO system 880 and JASCO system 987 instruments. Reversed-phase HPLC was carried out on a column of Deverosil ODS-15/30 and Deverosil ODS-5 (Nomura Chemical, Ltd.). Detection was by UV absorption at 280 or $205 \mathrm{~nm}$.

Propolis Material Brazilian propolis glue, which was obtained from the state of Minas Gerais in Brazil, was supplied by Dai Ki Kenko-kan Co., Ltd.

Extraction and Isolation Propolis $(450 \mathrm{~g})$ was extracted with $75 \%$ EtOH (1 l) at room temperature for $24 \mathrm{~h}$. After filtration, concentration of the $75 \%$ EtOH extract $(150 \mathrm{~g})$ under reduced pressure gave a crude material $(29.5 \mathrm{~g})$. It was partitioned with EtOAc- $\mathrm{H}_{2} \mathrm{O}$ to yield an EtOAc extract $(21.8$ $\mathrm{g})$, a $\mathrm{H}_{2} \mathrm{O}$ extract $(6.3 \mathrm{~g})$ and a residue $(1.4 \mathrm{~g})$. The EtOAc extract $(5.0 \mathrm{~g})$ was subjected to silica gel column chromatography with a $n$-hexane- $\mathrm{CHCl}_{3}$ $\mathrm{MeOH}$ gradient system to give eight fractions [fr. $1 n$-hexane eluate $(5.8 \mathrm{mg})$; fr. $2 \mathrm{n}$-hexane: $\mathrm{CHCl}_{3}=1: 1$ eluate $(5.8 \mathrm{mg}) ;$ fr. $3 \mathrm{CHCl}_{3}$ eluate $(1197.8 \mathrm{mg})$; fr. $4 \mathrm{CHCl}_{3}: \mathrm{MeOH}=9: 1$ eluate $(2354.3 \mathrm{mg})$; fr. $5 \mathrm{CHCl}_{3}$ : $\mathrm{MeOH}=8: 2$ eluate $(295.6 \mathrm{mg})$; fr. $6 \mathrm{CHCl}_{3}: \mathrm{MeOH}=1: 1$ eluate $(350.4$ $\mathrm{mg}$ ); fr. $7 \mathrm{MeOH}$ eluate $(295.9 \mathrm{mg})$; fr. $8 \mathrm{MeOH}+1 \% \mathrm{AcOH}$ eluate $(669.4 \mathrm{mg})]$

Fraction $3\left[\mathrm{CHCl}_{3}\right.$ eluate] $(1197.8 \mathrm{mg})$ was repeatedly rechromatographed by preparative HPLC [ODS: $2 \% \mathrm{AcOH}$ in $\mathrm{CH}_{3} \mathrm{CN}-\mathrm{H}_{2} \mathrm{O}, 1 \% \mathrm{AcOH}$ in $\mathrm{MeOH}-\mathrm{H}_{2} \mathrm{O}$ (UV $280 \mathrm{~nm}$ ) or $\mathrm{CH}_{3} \mathrm{CN}-\mathrm{H}_{2} \mathrm{O}, \mathrm{MeOH}-\mathrm{H}_{2} \mathrm{O}$ (UV $205 \mathrm{~nm}$ )] to furnish compounds $2(2.5 \mathrm{mg}), 6(3.0 \mathrm{mg}), 7(3.7 \mathrm{mg}), 8(12.5 \mathrm{mg}), 9(4.6$ $\mathrm{mg}), 10(4.1 \mathrm{mg}), 11(2.1 \mathrm{mg}), 12(2.5 \mathrm{mg}), 16(3.1 \mathrm{mg})$ and $23(5.9 \mathrm{mg})$. Fraction $4\left[\mathrm{CHCl}_{3}: \mathrm{MeOH}=9: 1\right.$ eluate] $(2.0 \mathrm{~g})$ was subjected to reversedphase chromatography by preparative HPLC, using a gradient solvent of $2 \%$ $\mathrm{AcOH}$ in $\mathrm{CH}_{3} \mathrm{CN}-\mathrm{H}_{2} \mathrm{O}(2: 8)$ to $(8: 2)$, to give 76 fractions. These were rechromatographed by preparative HPLC [ODS: $2 \% A c O H$ in $\mathrm{CH}_{3} \mathrm{CN}-\mathrm{H}_{2} \mathrm{O}$ and $1 \% \mathrm{AcOH}$ in $\left.\mathrm{MeOH}-\mathrm{H}_{2} \mathrm{O}(\mathrm{UV} 280 \mathrm{~nm})\right]$ to give compounds $1(1.1 \mathrm{mg})$, $3(2.6 \mathrm{mg}), 4(2.6 \mathrm{mg}), 5(3.1 \mathrm{mg}), 13(1.0 \mathrm{mg}), 14(2.7 \mathrm{mg}), 15(14.5 \mathrm{mg}), 17$ $(3.9 \mathrm{mg}), 18(1.8 \mathrm{mg}), 19(4.1 \mathrm{mg}), 20(1.7 \mathrm{mg}), 21(4.8 \mathrm{mg}), 22(3.0 \mathrm{mg}), 23$ $(7.0 \mathrm{mg})$ and $24(2.1 \mathrm{mg})$. The known compounds were identified by comparison of spectral data and/or specific optical rotation with reported values and/or authentic samples and chemical reactions.

Compound 18 Amorphous powder. FAB-MS $m / z: 329[\mathrm{M}+\mathrm{H}]^{+}$. UV $\lambda_{\max }^{\mathrm{MeOH}} \mathrm{nm}(\log \varepsilon): 224$ (4.36), 290 (4.24), 311 (4.26). IR (KBr): $1688 \mathrm{~cm}^{-1}$. 'H-NMR ( $\left.\mathrm{CD}_{3} \mathrm{OD}\right) \delta: 1.98\left(2 \mathrm{H}, \mathrm{dt}, J=7.5,6.5 \mathrm{~Hz}, \mathrm{H}-8^{\prime}\right), 2.66(2 \mathrm{H}, \mathrm{t}$ $\left.J=7.5 \mathrm{~Hz}, \mathrm{H}-7^{\prime}\right), 3.83(3 \mathrm{H}, \mathrm{s}, \mathrm{OMe}), 4.16\left(2 \mathrm{H}, \mathrm{t}, J=6.5 \mathrm{~Hz}, \mathrm{H}-9^{\prime}\right), 6.33(1 \mathrm{H}$, d, $J=16.0 \mathrm{~Hz}, \mathrm{H}-8), 6.64\left(1 \mathrm{H}, \mathrm{dd}, J=8.5,1.5 \mathrm{~Hz}, \mathrm{H}-6^{\prime}\right), 6.71(1 \mathrm{H}, \mathrm{d}, J=$ $\left.8.5 \mathrm{~Hz}, \mathrm{H}-5^{\prime}\right), 6.78\left(1 \mathrm{H}, \mathrm{d}, J=1.5 \mathrm{~Hz}, \mathrm{H}-2^{\prime}\right), 6.81(2 \mathrm{H}, \mathrm{d}, J=8.5 \mathrm{~Hz}, \mathrm{H}-3,5)$, $7.46(2 \mathrm{H}, \mathrm{d}, J=8.5 \mathrm{~Hz}, \mathrm{H}-2,6), 7.58(1 \mathrm{H}, \mathrm{d}, J=16.0 \mathrm{~Hz}, \mathrm{H}-7) .{ }^{13} \mathrm{C}-\mathrm{NMR}$ $\left(\mathrm{CD}_{3} \mathrm{OD}\right) \delta: 31.8\left(\mathrm{C}-8^{\prime}\right), 32.7\left(\mathrm{C}-7^{\prime}\right), 56.4(\mathrm{OMe}), 64.6\left(\mathrm{C}-9^{\prime}\right), 113.2\left(\mathrm{C}-2^{\prime}\right)$, $115.2\left(\mathrm{C}-5^{\prime}\right), 115.8$ (C-8), 116.8 (C-3, 5), 121.9 (C-6'), 127.2 (C-1), 131.2 $(\mathrm{C}-2,6), 133.4\left(\mathrm{C}-1^{\prime}\right), 145.7\left(\mathrm{C}-4^{\prime}\right), 146.5(\mathrm{C}-7), 148.9\left(\mathrm{C}-3^{\prime}\right), 161.3(\mathrm{C}-4)$, $168.6(\mathrm{C}-9)$.

Compound 19 Amorphous powder. FAB-MS $m / z: 394[\mathrm{M}]^{+}$. UV $\lambda_{\max }^{\mathrm{MeOH}}$ $\mathrm{nm}(\log \varepsilon): 211(4.43), 226(4.38), 311$ (4.59). IR (KBr): 1688, $1682 \mathrm{~cm}^{-1}$ $[\alpha]_{\mathrm{D}}^{25}-16.7^{\circ}(c=0.22, \mathrm{MeOH}) .{ }^{1} \mathrm{H}-\mathrm{NMR}\left(\mathrm{CD}_{3} \mathrm{OD}\right) \delta: 1.05(3 \mathrm{H}, \mathrm{d}, J=7.0$ $\mathrm{Hz}, \mathrm{H}-13), 2.23$ (1H, m, H-11), 3.11 (1H, dd, $J=16.0,8.0 \mathrm{~Hz}, \mathrm{Ha}-3), 3.35$ $(1 \mathrm{H}, \mathrm{dd}, J=16.0,4.5 \mathrm{~Hz}, \mathrm{Hb}-3), 4.22(2 \mathrm{H}, \mathrm{m}, \mathrm{H}-12), 4.85(1 \mathrm{H}, \mathrm{m}, \mathrm{H}-2), 6.30$ $(1 \mathrm{H}, \mathrm{d}, J=16.0 \mathrm{~Hz}, \mathrm{H}-9), 6.33\left(1 \mathrm{H}, \mathrm{d}, J=16.0 \mathrm{~Hz}, \mathrm{H}-8^{\prime}\right), 6.74(1 \mathrm{H}, \mathrm{d}, J=$
$8.5 \mathrm{~Hz}, \mathrm{H}-7), 6.81\left(2 \mathrm{H}, \mathrm{d}, J=8.5 \mathrm{~Hz}, \mathrm{H}-3^{\prime}, 5^{\prime}\right), 7.32(1 \mathrm{H}$, br d, $J=8.5 \mathrm{~Hz}, \mathrm{H}-$ 6), $7.46\left(2 \mathrm{H}, \mathrm{d}, J=8.5 \mathrm{~Hz}, \mathrm{H}-2^{\prime}, 6^{\prime}\right), 7.47(1 \mathrm{H}, \mathrm{br} \mathrm{s}, \mathrm{H}-4), 7.56(1 \mathrm{H}, \mathrm{d}$, $J=16.0 \mathrm{~Hz}, \mathrm{H}-8), 7.61\left(1 \mathrm{H}, \mathrm{d}, J=16.0 \mathrm{~Hz}, \mathrm{H}-7^{\prime}\right) .{ }^{13} \mathrm{C}-\mathrm{NMR}\left(\mathrm{CD}_{3} \mathrm{OD}\right) \delta$ : 11.7 (C-13), 33.5 (C-3), 39.4 (C-11), 67.1 (C-12), 85.8 (C-2), 110.2 (C-7), 115.0 (C-8'), 116.0 (C-9), 116.8 (C-3', 5'), 125.5 (C-4), 127.1 (C-1'), 128.9 (C-5), 129.5 (C-3a), 130.6 (C-6), 131.2 (C-2', 6'), 145.9 (C-8), 146.7 (C-7'), $161.3\left(\mathrm{C}^{\prime} 4^{\prime}\right), 163.2$ (C-7a), 169.2 (C-9'), 172.0 (C-10).

Compound 20 Amorphous powder. FAB-MS $m / z: 297[\mathrm{M}+\mathrm{H}]^{+}$. UV $\lambda_{\max }^{\mathrm{MeOH}} \mathrm{nm}(\log \varepsilon): 205$ (4.26), $272(4.11) . \mathrm{IR}(\mathrm{KBr}): 1757,1686 \mathrm{~cm}^{-1} .{ }^{1} \mathrm{H}-$ NMR $\left(\mathrm{CDCl}_{3}\right) \delta: 2.90\left(2 \mathrm{H}, \mathrm{t}, J=7.5 \mathrm{~Hz}, \mathrm{H}-8^{\prime}\right), 3.08(2 \mathrm{H}, \mathrm{t}, J=7.5 \mathrm{~Hz}, \mathrm{H}-$ $\left.7^{\prime}\right), 6.40(1 \mathrm{H}, \mathrm{d}, J=16.0 \mathrm{~Hz}, \mathrm{H}-8), 7.05(2 \mathrm{H}, \mathrm{d}, J=7.5 \mathrm{~Hz}, \mathrm{H}-3,5), 7.24$ $7.34\left(5 \mathrm{H}, \mathrm{m}, \mathrm{H}-2^{\prime}, 3^{\prime}, 4^{\prime}, 5^{\prime}, 6^{\prime}\right), 7.54(2 \mathrm{H}, \mathrm{d}, J=7.5 \mathrm{~Hz}, \mathrm{H}-2,6), 7.71(1 \mathrm{H}$, d, $J=16.0 \mathrm{~Hz}, \mathrm{H}-9) .{ }^{13} \mathrm{C}-\mathrm{NMR}\left(\mathrm{CDCl}_{3}\right) \delta: 30.9\left(\mathrm{C}-7^{\prime}\right), 36.0\left(\mathrm{C}-8^{\prime}\right), 117.0$ (C-8), 122.2 (C-3, 5), $126.5\left(\mathrm{C}-4^{\prime}\right), 128.4\left(\mathrm{C}-2^{\prime}, 6^{\prime}\right), 128.6\left(\mathrm{C}-3^{\prime}, 5^{\prime}\right), 129.4$ (C-2, 6), 131.9 (C-1), 139.9 (C-1'), 147.0 (C-7), 152.0 (C-8), 171.1 (C-9), $172.3\left(\mathrm{C}-9^{\prime}\right)$

Compound 21 Amorphous powder. FAB-MS $m / z: 248[\mathrm{M}]^{+}$. UV $\lambda_{\max }^{\mathrm{MeOH}}$ nm ( $\log \varepsilon): 202$ (4.19), 212 (4.14), 225 (4.06), 294 (4.17), 302 (4.16). IR $(\mathrm{KBr}): 1682 \mathrm{~cm}^{-1} \cdot[\alpha]_{\mathrm{D}}^{25}+1.35^{\circ}(c=0.22, \mathrm{MeOH}){ }^{1} \mathrm{H}-\mathrm{NMR}\left(\mathrm{CD}_{3} \mathrm{OD}\right) \delta$ : 1.26 (3H, s, H-13), 1.34 (3H, s, H-12), $2.75(1 \mathrm{H}, \mathrm{dd}, J=16.5,7.0 \mathrm{~Hz}, \mathrm{Ha}-4)$, $3.04(1 \mathrm{H}, \mathrm{dd}, J=16.5,5.0 \mathrm{~Hz}, \mathrm{Hb}-4), 3.78(1 \mathrm{H}, \mathrm{dd}, J=7.0,5.0 \mathrm{~Hz}, \mathrm{H}-3), 6.30$ $(1 \mathrm{H}, \mathrm{d}, J=16.0 \mathrm{~Hz}, \mathrm{H}-10), 6.76(1 \mathrm{H}, \mathrm{d}, J=8.0 \mathrm{~Hz}, \mathrm{H}-8), 7.32(1 \mathrm{H}, \mathrm{br} \mathrm{s}, \mathrm{H}-5)$, $7.34(1 \mathrm{H}, \mathrm{br} \mathrm{d}, J=8.0 \mathrm{~Hz}, \mathrm{H}-7), 7.58(1 \mathrm{H}, \mathrm{d}, J=16.0 \mathrm{~Hz}, \mathrm{H}-9) .{ }^{13} \mathrm{C}-\mathrm{NMR}$ ( $\left.\mathrm{CD}_{3} \mathrm{OD}\right) \delta: 21.4$ (C-13), 25.9 (C-12), 31.9 (C-4), 70.1 (C-3), $78.8(\mathrm{C}-2)$, 116.3 (C-10), 118.6 (C-8), 121.8 (C-4a), 128.1 (C-6), 128.5 (C-7), 131.5 (C5), 146.4 (C-9), 156.7 (C-8a), 171.0 (C-11).

Compound 22 Amorphous powder. FAB-MS $m / z: 231[\mathrm{M}+\mathrm{H}]^{+}$. UV $\lambda_{\max }^{\mathrm{MeOH}} \mathrm{nm}(\log \varepsilon): 203$ (4.30), 231 (4.09), $312(4.15)$. IR $(\mathrm{KBr}): 1682 \mathrm{~cm}^{-1}$. $[\alpha]_{\mathrm{D}}^{25}-0.74^{\circ}(c=0.14, \mathrm{MeOH}) .{ }^{1} \mathrm{H}-\mathrm{NMR}\left(\mathrm{CD}_{3} \mathrm{OD}\right) \delta: 1.79(3 \mathrm{H}, \mathrm{s}, \mathrm{H}-13)$, $2.78(1 \mathrm{H}$, dd, $J=13.5,8.0 \mathrm{~Hz}, \mathrm{Ha}-3), 2.91(1 \mathrm{H}, \mathrm{dd}, J=13.5,4.5 \mathrm{~Hz}, \mathrm{Hb}-3)$, $4.35(1 \mathrm{H}, \mathrm{dd}, J=8.0,4.5 \mathrm{~Hz}, \mathrm{H}-2), 4.77(1 \mathrm{H}, \mathrm{s}, \mathrm{Ha}-12), 4.85(1 \mathrm{H}, \mathrm{s}, \mathrm{Hb}-12)$, $6.27(1 \mathrm{H}, \mathrm{d}, J=16.0 \mathrm{~Hz}, \mathrm{H}-9), 6.79(1 \mathrm{H}, \mathrm{d}, J=8.5 \mathrm{~Hz}, \mathrm{H}-7), 7.31(1 \mathrm{H}, \mathrm{dd}$, $J=8.5,2.0 \mathrm{~Hz}, \mathrm{H}-6), 7.34(1 \mathrm{H}, \mathrm{d}, J=2.0 \mathrm{~Hz}, \mathrm{H}-4), 7.57(1 \mathrm{H}, \mathrm{d}, J=16.0 \mathrm{~Hz}$, $\mathrm{H}-8) .{ }^{13} \mathrm{C}-\mathrm{NMR}\left(\mathrm{CD}_{3} \mathrm{OD}\right) \delta: 18.1(\mathrm{C}-13), 38.1(\mathrm{C}-3), 76.4(\mathrm{C}-2), 111.3(\mathrm{C}-$ 12), 115.7 (C-9), 116.7 (C-7), 127.2 (C-5), 127.6 (C-3a), 129.1 (C-6), 132.8 (C-4), 146.8 (C-8), 148.7 (C-11), 159.4 (C-7a), 171.4 (C-10).

Diazomethane Methylation of Compound 22 A solution of 22 (2.8 $\mathrm{mg}$ ) in $\mathrm{MeOH}(1 \mathrm{ml})$ was methylated with an excess of $\mathrm{CH}_{2} \mathrm{~N}_{2}-\mathrm{Et}_{2} \mathrm{O}$ until the yellow color persisted. The solvent was removed under reduced pressure to furnish $22 \mathrm{a}(2.8 \mathrm{mg})$.

Methyl ester of 22 (22a) Amorphous powder. FAB-MS m/z: 245 $[\mathrm{M}+\mathrm{H}]^{+}$. UV $\lambda_{\max }^{\mathrm{MeOH}} \mathrm{nm}(\log \varepsilon): 203(4.30), 231$ (4.09), 312 (4.15). IR (KBr): $1688 \mathrm{~cm}^{-1} .{ }^{1} \mathrm{H}-\mathrm{NMR}\left(\mathrm{CDCl}_{3}\right) \delta: 1.82(3 \mathrm{H}, \mathrm{s}, \mathrm{H}-13), 2.78(1 \mathrm{H}, \mathrm{dd}$, $J=14.0,8.5 \mathrm{~Hz}, \mathrm{Ha}-3), 2.97(1 \mathrm{H}, \mathrm{dd}, J=14.0,4.0 \mathrm{~Hz}, \mathrm{Hb}-3), 3.79(\mathrm{OMe})$, $4.35(1 \mathrm{H}, \mathrm{dd}, J=8.5,4.0 \mathrm{~Hz}, \mathrm{H}-2), 4.84(1 \mathrm{H}, \mathrm{s}, \mathrm{Ha}-12), 4.95(1 \mathrm{H}, \mathrm{s}, \mathrm{Hb}-12)$, $6.32(1 \mathrm{H}, \mathrm{d}, J=16.0 \mathrm{~Hz}, \mathrm{H}-9), 6.87(1 \mathrm{H}, \mathrm{d}, J=8.5 \mathrm{~Hz}, \mathrm{H}-7), 7.37(1 \mathrm{H}, \mathrm{d}$, $J=2.0 \mathrm{~Hz}, \mathrm{H}-4), 7.40(1 \mathrm{H}, \mathrm{dd}, J=8.5,2.0 \mathrm{~Hz}, \mathrm{H}-6), 7.64(1 \mathrm{H}, \mathrm{d}, J=16.0 \mathrm{~Hz}$, $\mathrm{H}-8) .{ }^{13} \mathrm{C}-\mathrm{NMR}\left(\mathrm{CDCl}_{3}\right) \delta$ : $18.1(\mathrm{C}-13), 37.0(\mathrm{C}-3), 51.6(\mathrm{OMe}), 75.3(\mathrm{C}-2)$, 110.6 (C-12), 115.4 (C-9), 117.0 (C-7), 127.0 (C-5), 127.7 (C-3a), 128.5 (C6), 130.9 (C-4), 144.6 (C-8), 147.1 (C-11), 159.4 (C-7a), 167.7 (C-10).

Compound 23 Amorphous powder. FAB-MS $m / z: 299[\mathrm{M}+\mathrm{H}]^{+}$. UV $\lambda_{\max }^{\mathrm{MeOH}} \mathrm{nm}(\log \varepsilon): 218(4.16), 232$ (4.11), 303 (4.14). IR (KBr): $1682 \mathrm{~cm}^{-1}$. $[\alpha]_{\mathrm{D}}^{25}+2.25^{\circ}(c=0.37, \mathrm{MeOH}) .{ }^{1} \mathrm{H}-\mathrm{NMR}\left(\mathrm{CDCl}_{3}\right) \delta: 1.73\left(3 \mathrm{H}, \mathrm{s}, \mathrm{H}-5^{\prime}\right)$, $1.78\left(3 \mathrm{H}, \mathrm{s}, \mathrm{H}-4^{\prime}\right), 1.82(3 \mathrm{H}, \mathrm{s}, \mathrm{H}-13), 2.81(1 \mathrm{H}, \mathrm{d}, J=12.5 \mathrm{~Hz}, \mathrm{Ha}-3), 2.97$ $(1 \mathrm{H}, \mathrm{dd}, J=12.5,8.5 \mathrm{~Hz}, \mathrm{Hb}-3), 3.36\left(2 \mathrm{H}, \mathrm{d}, J=7.0 \mathrm{~Hz}, \mathrm{H}-1^{\prime}\right), 4.40(1 \mathrm{H}, \mathrm{d}$, $J=8.5 \mathrm{~Hz}, \mathrm{H}-2), 4.90(1 \mathrm{H}, \mathrm{s}, \mathrm{Ha}-12), 5.01(1 \mathrm{H}, \mathrm{s}, \mathrm{Hb}-12), 5.33(1 \mathrm{H}, \mathrm{br} \mathrm{t}, \mathrm{H}-$ $\left.2^{\prime}\right), 6.26(1 \mathrm{H}, \mathrm{d}, J=16.0 \mathrm{~Hz}, \mathrm{H}-9), 7.11(1 \mathrm{H}, \mathrm{br} \mathrm{s}, \mathrm{H}-4), 7.25(1 \mathrm{H}, \mathrm{br} \mathrm{s}, \mathrm{H}-6)$, $7.68(1 \mathrm{H}, \mathrm{d}, J=16.0 \mathrm{~Hz}, \mathrm{H}-8) .{ }^{13} \mathrm{C}-\mathrm{NMR}\left(\mathrm{CDCl}_{3}\right) \delta: 17.8\left(\mathrm{C}-5^{\prime}\right), 18.1(\mathrm{C}-$ 13), $25.8\left(\mathrm{C}-4^{\prime}\right), 28.7\left(\mathrm{C}-1^{\prime}\right), 38.1$ (C-3), 78.1 (C-2), 111.4 (C-12), 113.8 (C9), 121.9 (C-2'), 125.8 (C-3a), 126.0 (C-5), 128.9 (C-6), 129.8 (C-4), 130.2 (C-7), 133.4 (C-3'), 146.3 (C-11), 147.3 (C-8), 156.5 (C-7a), 171.9 (C-10).

Compound 24 Amorphous powder. FAB-MS m/z: $447[\mathrm{M}-\mathrm{H}]^{+}$. UV $\lambda_{\max }^{\mathrm{MeOH}} \mathrm{nm}(\log \varepsilon): 204$ (4.26), 208 (4.25), 232 (4.06), $305(4.05)$. IR $(\mathrm{KBr})$ : $1720,1686 \mathrm{~cm}^{-1}$. 'H-NMR $\left(\mathrm{CDCl}_{3}\right) \delta: 1.73\left(3 \mathrm{H}, \mathrm{s}, \mathrm{H}-5^{\prime}\right), 1.79(6 \mathrm{H}, \mathrm{s}, \mathrm{H}-$ $\left.4^{\prime \prime}, 5^{\prime \prime}\right), 2.67\left(2 \mathrm{H}, \mathrm{t}, J=7.5 \mathrm{~Hz}, \mathrm{H}-8^{\prime \prime \prime}\right), 2.96\left(2 \mathrm{H}, \mathrm{t}, J=7.5 \mathrm{~Hz}, \mathrm{H}-7^{\prime \prime \prime}\right), 3.36(4 \mathrm{H}$, br s, H-1' $\left.1^{\prime \prime}\right), 4.52\left(2 \mathrm{H}, \mathrm{s}, \mathrm{H}-4^{\prime}\right), 5.29\left(1 \mathrm{H}, \mathrm{br} \mathrm{t}, \mathrm{H}-2^{\prime \prime}\right), 5.60\left(1 \mathrm{H}, \mathrm{br} \mathrm{t}, \mathrm{H}-2^{\prime}\right)$, $6.31(1 \mathrm{H}, \mathrm{d}, J=16.0 \mathrm{~Hz}, \mathrm{H}-8), 7.18-7.20\left(5 \mathrm{H}, \mathrm{m}, \mathrm{H}-2,6,2^{\prime \prime \prime}, 4^{\prime \prime \prime}, 6^{\prime \prime \prime}\right), 7.25$ $\left(2 \mathrm{H}, \mathrm{m}, \mathrm{H}-3^{\prime \prime \prime}, 5^{\prime \prime \prime}\right), 7.68(1 \mathrm{H}, \mathrm{d}, J=16.0 \mathrm{~Hz}, \mathrm{H}-7) .{ }^{13} \mathrm{C}-\mathrm{NMR}\left(\mathrm{CDCl}_{3}\right) \delta: 14.0$ (C-5'), $17.9\left(\mathrm{C}-5^{\prime \prime}\right), 25.8\left(\mathrm{C}-4^{\prime \prime}\right), 28.5\left(\mathrm{C}-1^{\prime}\right), 30.1\left(\mathrm{C}-1^{\prime \prime}\right), 31.0\left(\mathrm{C}-7^{\prime \prime \prime}\right), 35.9$ (C-8"'), $69.8\left(\mathrm{C}-4^{\prime}\right), 114.5(\mathrm{C}-8), 121.0\left(\mathrm{C}-2^{\prime \prime}\right), 126.2$ (C-1, 2', 4"'), 127.0$128.6(\mathrm{C}-2,3,5,6), 128.3\left(\mathrm{C}-2^{\prime \prime \prime}, 6^{\prime \prime \prime}\right), 128.5\left(\mathrm{C}-3^{\prime \prime \prime}, 5^{\prime \prime \prime}\right), 132.3\left(\mathrm{C}-3^{\prime}\right), 136.1$ (C-3"), 140.4 (C-1"'), 146.9 (C-7), 155.2 (C-4), 171.0 (C-9), 172.8 (C-9"'). 
Acknowledgments We thank Dr. Mitsuo Uchida and the staff of the Central Analytical Laboratory of this University for the measurement of mass spectra.

\section{References and Note}

1) Part I: Tazawa S., Warashina T., Noro T., Miyase T., Chem. Pharm. Bull., 46, 1477-1479 (1998).

2) Farkas L.,Gabor M., Kallay F. (ed.), "Flavonoids and Bioflavonoids current Research Trends," Elsevier Scientific Publishing Co., New York, 1977.

3) Grande M., Piera F., Cuena A., Torres P., Bellido I. S., Planta Med., 51, 414-419(1985).

4) Manez S., Paya M., Terencio C., Villar A., Planta Med., 54, 187-188 (1988).

5) Merfort I., Wendisch D., Planta Med., 53, 434-437 (1987).

6) Shima K., Hisada S., Inagaki 1., Yakugaku Zasshi, 92, 1410-1414 (1972); Venkatasubbaiah P., Chilton W. S., J. Nat. Prod., 54, 12931297 (1991).

7) Fang J.-M., Hsu K.-C., Cheng Y.-S., Phytochemistry, 28, 1173-1175 (1989).
8) Bastard J., Duc D. K., Fetizon M., Francis M. J., Grant P. K., Weavers R.T., Kaneko C., Baddeley G. V., Bernassau J. M., Burfitt I. R., Wovkulich P. M., Wenkert E., J. Nat. Prod., 47, 592-599 (1984).

9) Ohmoto T., Kanatani K., Yamaguchi K., Chem. Pharm. Bull., 35, 229-234 (1987).

10) Valcic S., Montenegro G., Timmermann B. N., J. Nat. Prod., 61, 771-775 (1998).

11) Brown G. D., Phytochemistry, 35, 1037-1042 (1994); Labbe C., Rovirosa J., Faini F., Mahu M., San-Martin A., Castillo M., J. Nat. Prod. 49, 517-518(1986).

12) Schmitt A., Telikepalli H., Mitscher L. A., Phytochemistry, 30, 35693570 (1991).

13) Kitagawa I., Fukuda Y., Yoshihara M., Yamahara J., Yoshikawa M., Chem. Pharm. Bull., 31, 352-355 (1983).

14) Aga H., Shibuya T., Sugimoto T., Kurimoto M., Nakajima S., Biosci. Biotech. Biochem., 58, 945-946 (1994).

15) Marcucci M. C., Apidologie, 26, 83-99 (1995).

16) Bohlmann F., Knauf W., King R. M., Robinson H., Phytochemistry, 18, 1011-1014 (1979); Bankova V., Marcucci M. C., Nikolova N., Kujumgiev A., Popov S., Z. Naturforsch, 51c, 277-280 (1996). 